\title{
Dynamics of dislocation densities in a bounded channel. Part II: existence of weak solutions to a singular Hamilton-Jacobi/parabolic strongly coupled system
}

\author{
H. Ibrahim *, M. Jazar ${ }^{1}$, R. Monneau *
}

November 15, 2018

\begin{abstract}
We study a strongly coupled system consisting of a parabolic equation and a singular Hamilton-Jacobi equation in one space dimension. This system describes the dynamics of dislocation densities in a material submitted to an exterior applied stress. Our system is a natural extension of that studied in [15] where the applied stress was set to be zero. The equations are written on a bounded interval with Dirichlet boundary conditions and require special attention to the boundary. We prove a result of global existence of a solution. The method of the proof consists in considering first a parabolic regularization of the full system, and then passing to the limit. For this regularized system, a result of global existence and uniqueness of a solution has been given in [16]. We show some uniform bounds on this solution which uses in particular an entropy estimate for the densities.
\end{abstract}

AMS Classification: $70 \mathrm{H} 20,49 \mathrm{~L} 25,54 \mathrm{C} 70,46 \mathrm{E} 30$.

Key words: Hamilton-Jacobi equations, viscosity solutions, entropy, Orlicz spaces, parabolic equations.

\section{Introduction}

\subsection{Physical motivation and setting of the problem}

In [12], Groma, Czikor and Zaiser have proposed a model describing the dynamics of dislocation densities. Dislocations are defects in crystals that move when a stress field is applied on the material. These defects are one of the main explanations of the elastoviscoplasticity behavior of metals (see [8] and [9] for various models relating dislocations and elastoviscoplastic properties of metals). This model has been introduced in order to describe the possible accumulation of dislocations on the boundary layer of a bounded channel. Dislocations are distinguished by the sign of their Burgers vector $\pm \vec{b}$ (see [13] for a description of the Burgers vector). More precisely, let us call $\theta^{+}$and $\theta^{-}$, the densities of the positive and negative dislocations respectively. For

$$
x \in I:=(-1,1),
$$

${ }^{*}$ Cermics, Paris-Est-ENPC, ParisTech, 6 et 8 avenue Blaise Pascal, Cité Descartes Champs-sur-Marne, 77455 Marne-la-Vallée Cedex 2, France. E-mails: ibrahim@cermics.enpc.fr, monneau@cermics.enpc.fr.

${ }^{1}$ M. Jazar, LaMA-Liban, Lebanese University, P.O. Box 826, Tripoli Liban, mjazar@ul.edu.lb.

The second author is supported by a grant from the Lebanese University. 
and $t \in(0, T)$, for some time $T>0$, the non-negative quantities $\theta^{+}(x, t)$ and $\theta^{-}(x, t)$ are governed by the following system (see [12]):

$$
\left\{\begin{array}{l}
\theta_{t}^{+}=\left[\left(\frac{\theta_{x}^{+}-\theta_{x}^{-}}{\theta^{+}+\theta^{-}}-\tau\right) \theta^{+}\right]_{x} \quad \text { in } \quad I \times(0, T), \\
\theta_{t}^{-}=\left[-\left(\frac{\theta_{x}^{+}-\theta_{x}^{-}}{\theta^{+}+\theta^{-}}-\tau\right) \theta^{-}\right]_{x} \quad \text { in } \quad I \times(0, T),
\end{array}\right.
$$

where $\tau$ is the applied stress field which is assumed to be constant. Here the term $\tau_{b}=\frac{\theta_{x}^{+}-\theta_{x}^{-}}{\theta^{+}+\theta^{-}}$is called the back stress and can be interpreted as the contribution to the stress of the short-range interactions between dislocations. If $\tau=0$ and $\theta^{-}=0$ (resp. $\theta^{+}=0$ ) then $\theta^{+}$(resp. $\theta^{-}$) satisfies the usual heat equation. More generally, the back stress is proportional to the gradient of the effective dislocation density $\theta^{+}-\theta^{-}$, with a diffusion coefficient which is $\frac{1}{\theta^{+}+\theta^{-}}$. In fact, system (1.1) is a model for a $2 \mathrm{D}$ channel with coordinates $(x, y)$ that is invariant in the $y$-direction (see Figure 1). The channel is bounded by walls that are impenetrable

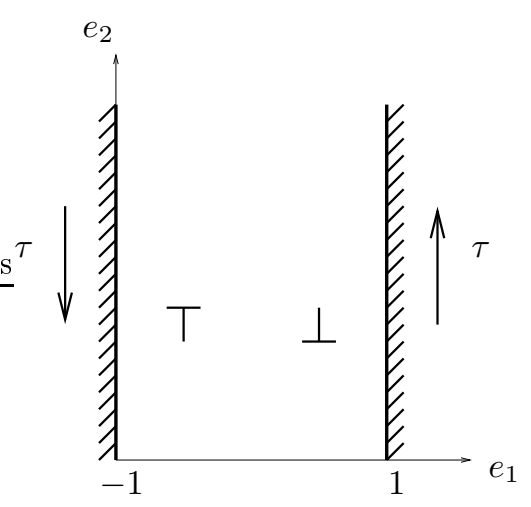

Figure 1: Geometry of the crystal.

by dislocations (i.e., the plastic deformation in the walls is zero). In this case the boundary conditions are represented by the zero flux condition, i.e.

$$
\frac{\theta_{x}^{+}-\theta_{x}^{-}}{\theta^{+}+\theta^{-}}-\tau=0, \quad \text { at } \quad x= \pm 1
$$

For related literature, let us mention the work of Groma-Balogh [11], where the back stress was neglected. For the model described in [11], we refer the reader to $[5,6]$ for a one-dimensional mathematical and numerical study, and to [4] for a two-dimensional existence result. The special case $\tau=0$ for system (1.1) has been studied in [15], where a result of existence and uniqueness has been proved. In the present paper we study the case for general constant $\tau$.

\subsection{Setting of the problem}

We consider an integrated form of (1.1) and we let

$$
\rho_{x}^{ \pm}=\theta^{ \pm}, \quad \rho=\rho^{+}-\rho^{-} \quad \text { and } \quad \kappa=\rho^{+}+\rho^{-},
$$

to obtain, for special values of the constants of integration, the following system in terms of $\rho$ and $\kappa$ :

$$
\begin{cases}\kappa_{t} \kappa_{x}=\rho_{t} \rho_{x} & \text { on } \quad I \times(0, T) \\ \rho_{t}=\rho_{x x}-\tau \kappa_{x} & \text { on } \quad I \times(0, T),\end{cases}
$$


with the initial conditions:

$$
\kappa(x, 0)=\kappa^{0}(x) \text { and } \quad \rho(x, 0)=\rho^{0}(x) .
$$

To formulate heuristically the boundary conditions at the walls located at $x= \pm 1$, we first suppose that $\kappa_{x} \neq 0$ at $x= \pm 1$. We recall that the dislocation fluxes at the walls must be zero, which require (1.2). Rewriting system (1.3) in terms of $\rho, \kappa$ and $\Phi=\frac{\theta_{x}^{+}-\theta_{x}^{-}}{\theta^{+}+\theta^{-}}-\tau$, we get

$$
\left\{\begin{array}{l}
\kappa_{t}=\rho_{x} \Phi \\
\rho_{t}=\kappa_{x} \Phi .
\end{array}\right.
$$

From (1.2) and (1.5), we deduce that

$$
\rho_{t}(x, .)=\kappa_{t}(x, .)=0 \quad \text { for } \quad x= \pm 1 .
$$

In this case, we consider the following boundary conditions:

$$
\left\{\begin{array}{lll}
\kappa(x, .)=x & \text { for } & x= \pm 1 \\
\rho(x, .)=0 & \text { for } & x= \pm 1
\end{array}\right.
$$

where we have taken the zero normalization for $\rho$ on the boundary of the interval.

The non-negativity of $\theta^{ \pm} \geq 0$ reduces in terms of $\rho$ and $\kappa$ to the following condition:

$$
\kappa_{x} \geq\left|\rho_{x}\right|,
$$

and hence a natural assumption to be considered concerning the initial conditions $\rho^{0}$ and $\kappa^{0}$ is to satisfy

$$
\kappa_{x}^{0} \geq\left|\rho_{x}^{0}\right| \quad \text { on } \quad I .
$$

As indicated above, problem (1.3), (1.4) and (1.7), in the case $\tau=0$, has been studied in [15], where a result of existence and uniqueness is given using the viscosity/entropy solution framework. Let us just mention that in this situation, system (1.3) becomes decoupled and easier to be handled.

\subsection{Statement of the main result}

Remark that the first equation of system (1.3) can be formally rewritten $\kappa_{t}=\rho_{t} \rho_{x} / \kappa_{x}$ which shows the singularity as $\kappa_{x}$ goes to zero. Nevertheless, for this system we have the following result.

Theorem 1.1 (Global existence of a solution). Let $\rho^{0}, \kappa^{0} \in C^{\infty}(\bar{I})$ satisfying (1.9),

$$
\kappa^{0}( \pm 1)= \pm 1, \quad \rho^{0}( \pm 1)=0
$$

and the additional conditions:

$$
D_{x}^{s} \rho^{0}( \pm 1)=D_{x}^{s} \kappa^{0}( \pm 1)=0, \quad s=1,2 .
$$

Then there exists $(\rho, \kappa)$ such that for every $T>0$ :

$$
(\rho, \kappa) \in(C(\bar{I} \times[0, T]))^{2} \quad \text { and } \quad \rho \in C^{1}(I \times(0, T)),
$$

is a solution of (1.3), (1.4) and (1.7). Moreover, this solution satisfies (1.8) in the distributional sense, i.e.

$$
\kappa_{x} \geq\left|\rho_{x}\right| \quad \text { in } \quad \mathcal{D}^{\prime}(I \times(0, T)) .
$$

However, the solution has to be interpreted in the following sense: 
1. $\kappa$ is a viscosity solution of $\kappa_{t} \kappa_{x}=\rho_{t} \rho_{x}$ in $I_{T}:=I \times(0, T)$,

2. $\rho$ is a distributional solution of $\rho_{t}=\rho_{x x}-\tau \kappa_{x}$ in $I_{T}$,

3. the initial and boundary conditions are satisfied pointwisely.

Remark 1.2 The $C^{\infty}$ regularity of $\rho^{0}$ and $\kappa^{0}$, together with (1.11) seems to be essentially technical and are related to an existence result for a regularized system (see Section 3, Theorem 3.1), that we use to prove Theorem 1.1.

\subsection{Organization of the paper}

This paper is organized as follows: in Section 2, we present the strategy of the proof. In Section 3, we present the tools needed throughout this work. This includes some miscellaneous results for parabolic equations, a brief recall to the definition and the stability result of viscosity solutions, and a brief recall to Orlicz spaces. In Section 4, we show how to choose the regularized solution. An entropy inequality used to determine some uniform bounds on the regularized solution is presented in Section 5. Further uniform bounds and convergence arguments are done in Section 6. Section 7 is devoted to the proof of our main result: Theorem 1.1. In Section 8, some numerical simulations related to our physical model are presented. Finally, Section 9 is an appendix where we show the proofs of some technical results.

\section{Strategy of the proof}

The main difficulty we have to face is to work with the equation

$$
\kappa_{t} \kappa_{x}=\rho_{t} \rho_{x}
$$

Since $\rho$ solves itself a parabolic equation (see the second equation of (1.3)), we expect enough regularity on $\rho$ (indeed $\rho$ is $C^{1}$ ), and then we need a framework where the equation involving $\kappa$ is stable under approximation. This property is naturally satisfied in the framework of viscosity solutions (see for instance [2] and the references therein). Then, assuming $\kappa_{x} \geq 0$, we interpret $\kappa$ as the viscosity solution of (2.1). Assuming (1.9), we will indeed show that

$$
M:=\kappa_{x}-\left|\rho_{x}\right| \geq 0
$$

This is formally true because $M$ satisfies:

$$
M_{t}=b M_{x}+c M
$$

with

$$
b=\tau \operatorname{sgn}\left(\rho_{x}\right)-\frac{\rho_{x} \rho_{x x}}{\kappa_{x}^{2}}, \quad c=\frac{\rho_{x x}^{2}}{\kappa_{x}^{2}}-\frac{\rho_{x x x} \operatorname{sgn}\left(\rho_{x}\right)}{\kappa_{x}},
$$

where for suitable boundary conditions, we can (again formally) see that $M \geq 0$. In order to justify the computations on $M$, we modify the system and we consider the following parabolic regularization for $\varepsilon>0$ small enough:

$$
\begin{cases}\kappa_{t}^{\varepsilon}=\varepsilon \kappa_{x x}^{\varepsilon}+\frac{\rho_{x}^{\varepsilon} \rho_{x x}^{\varepsilon}}{\kappa_{x}^{\varepsilon}}-\tau \rho_{x}^{\varepsilon} & \text { in } \quad I \times(0, \infty) \\ \rho_{t}^{\varepsilon}=(1+\varepsilon) \rho_{x x}^{\varepsilon}-\tau \kappa_{x}^{\varepsilon} & \text { in } I \times(0, \infty),\end{cases}
$$

with the initial conditions:

$$
\kappa^{\varepsilon}(x, 0)=\kappa^{0, \varepsilon}(x), \quad \rho^{\varepsilon}(x, 0)=\rho^{0, \varepsilon}(x),
$$


where $\kappa^{0, \varepsilon}$ and $\rho^{0, \varepsilon}$ are some regularizations of $\kappa^{0}$ and $\rho^{0}$ respectively, and the same boundary conditions:

$$
\left\{\begin{array}{lll}
\kappa^{\varepsilon}(x, .)=x & \text { for } & x= \pm 1 \\
\rho^{\varepsilon}(x, .)=0 & \text { for } & x= \pm 1
\end{array}\right.
$$

The system (2.2) formally reduces to (1.3) for $\varepsilon=0$. In fact, system (2.2), (2.3) and (2.4) has (under some conditions on the initial and boundary data) a unique smooth global solution (see [16, Theorem 1.1]) for $\alpha \in(0,1)$ :

$$
\left(\rho^{\varepsilon}, \kappa^{\varepsilon}\right) \in C^{3+\alpha, \frac{3+\alpha}{2}}(\bar{I} \times[0, \infty)) \cap C^{\infty}(\bar{I} \times(0, \infty)) .
$$

This result will be recalled in the forthcoming section (see Section 3, Theorem 3.1). The next step is to find some uniform bounds (independent of $\varepsilon$ ) on this solution; this is done in particular via:

(1) an entropy inequality shown to be valid for our regularized model (2.2);

(2) a bound on $\kappa_{t}^{\varepsilon}-\varepsilon \kappa_{x x}^{\varepsilon}$ uniformly in $\varepsilon$.

In fact, (1) guarantees the global uniform-in-time control of the modulus of continuity in space of our regularized solution, while (2) guarantees the local uniform-in-space control of the modulus of continuity in time. The entropy inequality can be easily understood. For instance, for $\varepsilon=0$ and $\tau=0$, we can formally check that the entropy of the dislocation densities

$$
\theta^{ \pm}=\frac{\kappa_{x} \pm \rho_{x}}{2}
$$

defined by:

$$
S(t)=\int_{I} \sum_{ \pm} \theta^{ \pm}(., t) \log \left(\theta^{ \pm}(., t)\right)
$$

satisfies:

$$
\frac{d S(t)}{d t}=-\int_{I} \frac{\left(\theta_{x}^{+}-\theta_{x}^{-}\right)^{2}}{\theta^{+}+\theta^{-}} \leq 0,
$$

therefore we get $S(t) \leq S(0)$ which controls the entropy uniformly in time. Finally, we need to pass to the limit $\varepsilon \rightarrow 0$ after multiplying the first equation of (2.2) by $\kappa_{x}^{\varepsilon}$. Having enough control on the regularized solutions, we can find a solution of the limit equation using in particular the stability of viscosity solutions of Hamilton-Jacobi equations. However, the passage to the limit in the second equation of (2.2) is done in the distributional sense.

\section{Tools: miscellaneous results on parabolic equations, viscosity so- lution, and Orlicz spaces}

\subsection{Miscellaneous results on parabolic equations}

We first fix some notations. Denote

$$
I_{T}:=I \times(0, T), \quad \overline{I_{T}}:=\bar{I} \times[0, T], \quad \partial^{p} I_{T}:=I \cup(\partial I \times[0, T]), \quad \text { and } \quad\|\cdot\|_{L^{p}(\Omega)}=\|\cdot\|_{p, \Omega} .
$$

Define the parabolic Sobolev space $W_{p}^{2,1}\left(I_{T}\right), 1<p<\infty$ by:

$$
W_{p}^{2,1}\left(I_{T}\right):=\left\{u \in L^{p}\left(I_{T}\right) ;\left(u_{t}, u_{x}, u_{x x}\right) \in\left(L^{p}\left(I_{T}\right)\right)^{3}\right\} .
$$

We start with a result of global existence and uniqueness of smooth solutions of the regularized system (2.2), with the initial and boundary conditions (2.3) and (2.4). 
Theorem 3.1 (Global existence for the regularized system, [16, Theorem 1.1]). Let $0<\alpha<1$ and $0<\varepsilon<1$. Let $\rho^{0, \varepsilon}, \kappa^{0, \varepsilon}$ satisfying:

$$
\begin{gathered}
\rho^{0, \varepsilon}, \kappa^{0, \varepsilon} \in C^{\infty}(\bar{I}), \quad \rho^{0, \varepsilon}( \pm 1)=0, \quad \text { and } \quad \kappa^{0, \varepsilon}( \pm 1)= \pm 1, \\
\left\{\begin{array}{lll}
(1+\varepsilon) \rho_{x x}^{0, \varepsilon}=\tau \kappa_{x}^{0, \varepsilon} & \text { on } \quad \partial I \\
(1+\varepsilon) \kappa_{x x}^{0, \varepsilon}=\tau \rho_{x}^{0, \varepsilon} & \text { on } \quad \partial I,
\end{array}\right.
\end{gathered}
$$

and

$$
\kappa_{x}^{0, \varepsilon}>\left|\rho_{x}^{0, \varepsilon}\right| \quad \text { on } \bar{I}
$$

Then there exists a unique global solution

$$
\left(\rho^{\varepsilon}, \kappa^{\varepsilon}\right) \in C^{3+\alpha, \frac{3+\alpha}{2}}(\bar{I} \times[0, \infty)) \cap C^{\infty}(\bar{I} \times(0, \infty)),
$$

of the system (2.2), (2.3) and (2.4). Moreover, this solution satisfies :

$$
\kappa_{x}^{\varepsilon}>\left|\rho_{x}^{\varepsilon}\right| \quad \text { on } \quad \bar{I} \times[0, \infty) .
$$

Remark 3.2 Conditions (3.2) are natural here. Indeed, the regularity (3.4) of the solution of equation (2.2) with boundary conditions (2.3) and (2.4) imply in particular condition (3.2).

Remark 3.3 (Uniform $L^{\infty}$ bound on $\rho^{\varepsilon}$ and $\kappa^{\varepsilon}$ ). We remark, from the boundary conditions (2.4) and from the inequality (3.5), that:

$$
\left\|\rho^{\varepsilon}\right\|_{L^{\infty}(\bar{I} \times[0, \infty))} \leq 1 \quad \text { and } \quad\left\|\kappa^{\varepsilon}\right\|_{L^{\infty}(\bar{I} \times[0, \infty))} \leq 1 .
$$

We now present two technical lemmas that will be used in the proof of Theorem 1.1. The proofs of these lemmas will be given in the Appendix.

Lemma 3.4 (Control of the modulus of continuity in time uniformly in $\varepsilon$ ). Let $p>3$, and $u^{\varepsilon} \in W_{p}^{2,1}\left(I_{T}\right)$. Suppose furthermore that the sequences

$$
\left(u^{\varepsilon}\right)_{\varepsilon} \quad \text { and } \quad\left(f^{\varepsilon}\right)_{\varepsilon}=\left(u_{t}^{\varepsilon}-\varepsilon u_{x x}^{\varepsilon}\right)_{\varepsilon}
$$

are locally bounded in $I_{T}$ uniformly for $\varepsilon \in(0,1)$. Then for every $V \subset \subset I_{T}$, there exist two constants $c>0$, $\varepsilon_{0}>0$ depending on $V$, and $0<\beta<1$ such that for all $0<\varepsilon<\varepsilon_{0}$ :

$$
\frac{\left|u^{\varepsilon}(x, t+h)-u^{\varepsilon}(x, t)\right|}{h^{\beta}} \leq c, \quad \forall(x, t),(x, t+h) \in V .
$$

Lemma 3.5 (An interior estimate for the heat equation). Let $a \in C^{\infty}\left(I_{T}\right) \cap L^{1}\left(I_{T}\right)$ satisfying:

$$
a_{t}=a_{x x} \quad \text { on } \quad I_{T},
$$

then for any $V \subset \subset I_{T}$, an open set, we have:

$$
\|a\|_{p, V} \leq c\|a\|_{1, I_{T}}, \quad \forall 1<p<\infty
$$

where $c=c(p, V)>0$ is a positive constant. 


\subsection{Viscosity solution: definition and stability result}

Let $\Omega \subset \mathbb{R}^{n}$ be an open domain, and consider the following Hamilton-Jacobi equation:

$$
H\left(x, u(x), D u(x), D^{2} u(x)\right)=0, \quad \forall x \in \Omega,
$$

where $H: \Omega \times \mathbb{R} \times \mathbb{R}^{n} \times M_{\text {sym }}^{n \times n} \rightarrow \mathbb{R}$ is a continuous mapping.

Definition 3.6 (Viscosity solution of Hamilton-Jacobi equations). A continuous function $u: \Omega \mapsto$ $\mathbb{R}$ is a viscosity sub-solution of (3.7) if for any $\phi \in C^{2}(\Omega ; \mathbb{R})$ and any local maximum $x_{0} \in \Omega$ of $u-\phi$, one has

$$
H\left(x_{0}, u\left(x_{0}\right), D \phi\left(x_{0}\right), D^{2} \phi\left(x_{0}\right)\right) \leq 0 .
$$

Similarly, $u$ is a viscosity super-solution of (3.7), if at any local minimum point $x_{0} \in \Omega$ of $u-\phi$, one has

$$
H\left(x_{0}, u\left(x_{0}\right), D \phi\left(x_{0}\right), D^{2} \phi\left(x_{0}\right)\right) \geq 0 .
$$

Finally, if $u$ is both a viscosity sub-solution and a viscosity super-solution, then $u$ is called a viscosity solution.

To get a "non-empty" and useful definition, it is usually assumed that $H$ is elliptic (see [2]). This notion of ellipticity will be indirectly used in Section 7. In fact, this definition is used for interpreting solutions of the first equation of (1.3) in the viscosity sense. This will be shown in Section 5. To be more precise, in the case where $\Omega=I_{T}$, we say that $u$ is a viscosity solution of the Dirichlet problem (3.7) with $u=\zeta \in C\left(\partial^{p} I_{T}\right)$ if:

(1) $u \in C\left(\overline{I_{T}}\right)$,

(2) $u$ is a viscosity solution of $(3.7)$ in $I_{T}$,

(3) $u=\zeta$ on $\partial^{p} I_{T}$.

For a better understanding of the viscosity interpretation of boundary conditions of Hamilton-Jacobi equations, we refer the reader to [2, Section 4.2]. We now state a stability result for viscosity solutions of Hamilton-Jacobi equations.

Theorem 3.7 (Stability of viscosity solutions, [2, Lemma 2.3]). Suppose that, for $\varepsilon>0, u^{\varepsilon} \in C(\Omega)$ is a viscosity sub-solution (resp. super-solution) of the equation

$$
H^{\varepsilon}\left(x, u^{\varepsilon}, D u^{\varepsilon}, D^{2} u^{\varepsilon}\right)=0 \quad \text { in } \quad \Omega,
$$

where $\left(H^{\varepsilon}\right)_{\varepsilon}$ is a sequence of continuous functions. If $u^{\varepsilon} \rightarrow u$ locally uniformly in $\Omega$ and if $H^{\varepsilon} \rightarrow H$ locally uniformly in $\Omega \times \mathbb{R} \times \mathbb{R}^{n} \times M_{\text {sym }}^{n \times n}$, then $u$ is a viscosity sub-solution (resp. super-solution) of the equation:

$$
H\left(x, u, D u, D^{2} u\right)=0 \quad \text { in } \quad \Omega .
$$

\subsection{Orlicz spaces: definition and properties}

We recall the definition of an Orlicz space and some of its properties (for details see [1]). A real valued function $\Psi:[0, \infty) \rightarrow \mathbb{R}$ is called a Young function if

$$
\Psi(t)=\int_{0}^{t} \psi(s) d s,
$$

where $\psi:[0, \infty) \rightarrow[0, \infty)$ satisfying:

- $\psi(0)=0, \psi>0$ on $(0, \infty), \psi(t) \rightarrow \infty$ as $t \rightarrow \infty$;

- $\psi$ is non-decreasing and right continuous at any point $s \geq 0$. 
Let $\Psi$ be a Young function. The Orlicz class $K_{\Psi}(I)$ is the set of equivalence classes of real-valued measurable functions $u$ on $I$ satisfying

$$
\int_{I} \Psi(|u(x)|) d x<+\infty
$$

Definition 3.8 (Orlicz spaces). The Orlicz space $L_{\Psi}(I)$ is the linear span of $K_{\Psi}(I)$ supplemented with the Luxemburg norm

$$
\|u\|_{L_{\Psi}(I)}=\inf \left\{k>0 ; \int_{I} \Psi\left(\frac{|u(x)|}{k}\right) \leq 1\right\}
$$

and with this norm, the Orlicz space is a Banach space.

The function

$$
\Phi(t)=\int_{0}^{t} \phi(s) d s, \quad \phi(s)=\sup _{\psi(t) \leq s} t,
$$

is called the complementary Young function of $\Psi$. An example of such pair of complementary Young functions is the following:

$$
\Psi(s)=(1+s) \log (1+s)-s \quad \text { and } \quad \Phi(s)=e^{s}-s-1 .
$$

We now state a lemma giving two useful properties of Orlicz spaces that will be used in the proof of Lemma 5.4 .

Lemma 3.9 (Norm control and Hölder inequality, [17]). If $u \in L_{\Psi}(I)$ for some Young function $\Psi$, then we have:

$$
\|u\|_{L_{\Psi}(I)} \leq 1+\int_{I} \Psi(|u(x)|) d x .
$$

Moreover, if $v \in L_{\Phi}(I), \Phi$ being the complementary Young function of $\Psi$, then we have the following Hölder inequality:

$$
\left|\int_{I} u v d x\right| \leq 2\|u\|_{L_{\Psi}(I)}\|v\|_{L_{\Phi}(I)}
$$

\section{The regularized problem}

As we have already mentioned, we will use a parabolic regularization of (1.3), and a result of global existence of this regularized system from [16] (see Theorem 3.1). In order to use this result, we need to give a special attention to the conditions on the initial data of the approximated system $\rho^{0, \varepsilon}$ and $\kappa^{0, \varepsilon}$ (see (3.1), (3.2) and (3.3)). This section aims to show how to choose the suitable initial data $\rho^{0, \varepsilon}$ and $\kappa^{0, \varepsilon}$ in order to benefit Theorem 3.1. Let $\rho^{0}$ and $\kappa^{0}$ be the functions given in Theorem 1.1. Set

$$
\rho^{0, \varepsilon}(x)=\frac{\rho^{0}(x)+\varepsilon \tau \phi(x)}{(1+\varepsilon)^{2}} \quad \text { and } \quad \kappa^{0, \varepsilon}(x)=\frac{\kappa^{0}(x)+\varepsilon x}{1+\varepsilon},
$$

with the function $\phi$ defined by:

$$
\phi(x)= \begin{cases}\frac{1}{4 \tau^{2}}\left[1-\cos \tau\left(x^{2}-1\right)\right] & \text { if } \tau \neq 0 \\ 0 & \text { if } \tau=0\end{cases}
$$

The function $\phi$ enjoys some properties that are shown in the following lemma.

Lemma 4.1 (Properties of $\phi$ )

The function $\phi$ given by (4.2) satisfies the following properties:

$$
\text { (P1) } \phi,\left.\phi^{\prime}\right|_{\partial I}=0
$$




$$
\begin{aligned}
& \text { (P2) }\left.\phi^{\prime \prime}\right|_{\partial I}=1 ; \\
& \text { (P3) }\left|\phi^{\prime}(x)\right|<1 /|\tau| \quad \text { for } \quad x \in \bar{I} .
\end{aligned}
$$

Proof. Straightforward computations.

Form the above lemma, and from the construction of $\rho^{0, \varepsilon}$ and $\kappa^{0, \varepsilon}$ (see (4.1)) together with the properties enjoyed by $\rho^{0}$ and $\kappa^{0}$ (see (1.10) and (1.11)), we write down some properties of $\rho^{0, \varepsilon}$ and $\kappa^{0, \varepsilon}$.

Lemma 4.2 (Properties of $\rho^{0, \varepsilon}$ and $\kappa^{0, \varepsilon}$ )

The functions $\rho^{0, \varepsilon}$ and $\kappa^{0, \varepsilon}$ given by (4.1), satisfy the following properties:

$$
\begin{aligned}
& \text { (P4) } \rho^{0, \varepsilon}( \pm 1)= \pm 1, \quad \text { and } \quad \kappa^{0, \varepsilon}( \pm 1)=0 \\
& \left.(P 5)(1+\varepsilon) \kappa_{x x}^{0, \varepsilon}\right|_{\partial I}=\left.\tau \rho_{x}^{0, \varepsilon}\right|_{\partial I} \quad \text { and }\left.\quad(1+\varepsilon) \rho_{x x}^{0, \varepsilon}\right|_{\partial I}=\left.\tau \kappa_{x}^{0, \varepsilon}\right|_{\partial I} \\
& \text { (P6) } \kappa_{x}^{0, \varepsilon} \geq\left|\rho_{x}^{0, \varepsilon}\right|+\frac{\varepsilon\left(1-|\tau|\left|\phi^{\prime}\right|\right)}{1+\varepsilon}>\left|\rho_{x}^{0, \varepsilon}\right| .
\end{aligned}
$$

Proof. Straightforward computations.

Remark 4.3 (The regularized solution $\left.\left(\rho^{\varepsilon}, \kappa^{\varepsilon}\right)\right)$. Properties (P4)-(P5)-(P6) of Lemma 4.2 implies condition (3.1)-(3.2)-(3.3) of Theorem 3.1. In this case, call

$$
\left(\rho^{\varepsilon}, \kappa^{\varepsilon}\right),
$$

the solution of (2.2), (2.3) and (2.4), given in Theorem 3.1, with the initial conditions

$$
\rho(x, 0)=\rho^{0, \varepsilon} \quad \text { and } \quad \kappa(x, 0)=\kappa^{0, \varepsilon},
$$

that are given by (4.1).

\section{$5 \quad$ Entropy inequality}

Proposition 5.1 (Entropy inequality). Let $\left(\rho^{\varepsilon}, \kappa^{\varepsilon}\right)$ be the regular solution given by (4.3). Define $\theta^{ \pm, \varepsilon}$ by:

$$
\theta^{ \pm, \varepsilon}=\frac{\kappa_{x}^{\varepsilon} \pm \rho_{x}^{\varepsilon}}{2}
$$

then the quantity $S(t)$ given by:

$$
S(t)=\int_{I} \sum_{ \pm} \theta^{ \pm, \varepsilon}(x, t) \log \theta^{ \pm, \varepsilon}(x, t) d x
$$

satisfies for every $t \geq 0$ :

$$
S(t) \leq S(0)+\frac{\tau^{2} t}{2} .
$$

Proof. From (3.5), we know that $\kappa_{x}^{\varepsilon}>\left|\rho_{x}^{\varepsilon}\right|$, hence $\theta^{ \pm, \varepsilon}>0$, and the term $\log \left(\theta^{ \pm, \varepsilon}\right)$ is well defined. Also from the regularity (3.4) of the solution $\left(\rho^{\varepsilon}, \kappa^{\varepsilon}\right)$, we know that $\theta^{ \pm, \varepsilon}(., t) \in C(\bar{I})$ for all $t \geq 0$, hence the term $S(t)$ is well defined. We derive system (2.2) with respect to $x$, and we write it in terms of $\theta^{ \pm, \varepsilon}$, we get:

$$
\left\{\begin{array}{l}
\theta_{t}^{+, \varepsilon}=\left[\left(\frac{\left(\theta^{+, \varepsilon}-\theta^{-, \varepsilon}\right)_{x}}{\theta^{+, \varepsilon}+\theta^{-, \varepsilon}}-\tau\right) \theta^{+, \varepsilon}+\varepsilon \theta_{x}^{+, \varepsilon}\right]_{x} \\
\theta_{t}^{-, \varepsilon}=\left[-\left(\frac{\left(\theta^{+, \varepsilon}-\theta^{-, \varepsilon}\right)_{x}}{\theta^{+, \varepsilon}+\theta^{-, \varepsilon}}-\tau\right) \theta^{-, \varepsilon}+\varepsilon \theta_{x}^{-, \varepsilon}\right]_{x} .
\end{array}\right.
$$


We first remark that:

$$
\pm\left(\frac{\left(\theta^{+, \varepsilon}-\theta^{-, \varepsilon}\right)_{x}}{\theta^{+, \varepsilon}+\theta^{-, \varepsilon}}-\tau\right) \theta^{ \pm, \varepsilon}+\varepsilon \theta_{x}^{ \pm, \varepsilon}=\frac{\kappa_{t}^{\varepsilon} \pm \rho_{t}^{\varepsilon}}{2}
$$

Since $\kappa_{t}^{\varepsilon}$ and $\rho_{t}^{\varepsilon}$ are zeros on $\partial I \times[0, \infty)$, then

$$
\left(\frac{\left(\theta^{+, \varepsilon}-\theta^{-, \varepsilon}\right)_{x}}{\theta^{+, \varepsilon}+\theta^{-, \varepsilon}}-\tau\right) \theta^{+, \varepsilon}+\varepsilon \theta_{x}^{+, \varepsilon}=-\left(\frac{\left(\theta^{+, \varepsilon}-\theta^{-, \varepsilon}\right)_{x}}{\theta^{+, \varepsilon}+\theta^{-, \varepsilon}}-\tau\right) \theta^{-, \varepsilon}+\varepsilon \theta_{x}^{-, \varepsilon}=0 \text { on } \partial I \times[0, \infty) .
$$

Using (5.5), we compute for $t \geq 0$ :

$$
\begin{aligned}
S^{\prime}(t) & =\sum_{ \pm} \int_{I} \theta_{t}^{ \pm, \varepsilon} \log \left(\theta^{ \pm, \varepsilon}\right)+\theta_{t}^{ \pm, \varepsilon} \\
& =\sum_{ \pm} \int_{I} \mp\left(\frac{\left(\theta^{+, \varepsilon}-\theta^{-, \varepsilon}\right)_{x}}{\theta^{+, \varepsilon}+\theta^{-, \varepsilon}}-\tau\right) \theta_{x}^{ \pm, \varepsilon}-\varepsilon \frac{\left(\theta_{x}^{ \pm, \varepsilon}\right)^{2}}{\theta^{ \pm, \varepsilon}} \\
& =\int_{I}-\frac{\left(\theta_{x}^{+, \varepsilon}-\theta_{x}^{-, \varepsilon}\right)^{2}}{\theta^{+, \varepsilon}+\theta^{-, \varepsilon}}+\tau\left(\theta_{x}^{+, \varepsilon}-\theta_{x}^{-, \varepsilon}\right)-\varepsilon\left(\frac{\left(\theta_{x}^{+, \varepsilon}\right)^{2}}{\theta^{+, \varepsilon}}+\frac{\left(\theta_{x}^{-, \varepsilon}\right)^{2}}{\theta^{-, \varepsilon}}\right)
\end{aligned}
$$

where we have integrated by parts in the second line. By Young's Inequality, we have:

$$
\left|\theta_{x}^{+, \varepsilon}-\theta_{x}^{-, \varepsilon}\right| \leq \frac{1}{|\tau|} \frac{\left(\theta_{x}^{+, \varepsilon}-\theta_{x}^{-, \varepsilon}\right)^{2}}{\theta^{+, \varepsilon}+\theta^{-, \varepsilon}}+\frac{|\tau|}{4}\left(\theta^{+, \varepsilon}+\theta^{-, \varepsilon}\right),
$$

and hence

$$
S^{\prime}(t) \leq \frac{\tau^{2}}{4} \int_{I}\left(\theta^{+, \varepsilon}+\theta^{-, \varepsilon}\right)
$$

Moreover, we have from (2.4), that

$$
\int_{I}\left(\theta^{+, \varepsilon}(., t)+\theta^{-, \varepsilon}(., t)\right)=\int_{I} \kappa_{x}^{\varepsilon}(., t)=\kappa^{\varepsilon}(1, t)-\kappa^{\varepsilon}(-1, t)=2
$$

and therefore

$$
S^{\prime}(t) \leq \frac{\tau^{2}}{2}
$$

Integrating the previous inequality from 0 to $t$, we get (5.3).

An immediate corollary of Proposition 5.1 is the following:

\section{Corollary 5.2 (Special control of $\kappa_{x}^{\varepsilon}$ )}

For all $t \geq 0$, we have:

$$
\int_{I} \kappa_{x}^{\varepsilon}(x, t) \log \left(\kappa_{x}^{\varepsilon}(x, t)\right) d x \leq S(0)+\frac{\tau^{2} t}{2}+2,
$$

where $S$ is given by (5.2).

The proof of Corollary 5.2 comes from the following inequality.

Lemma 5.3 For every $x, y>0$, we have:

$$
(x+y) \log (x+y) \leq x \log (x)+y \log (y)+x \log (2)+y .
$$


Proof. Direct computations.

Proof of Corollary 5.2. From (5.1), it follows that

$$
\kappa_{x}^{\varepsilon}=\theta^{+, \varepsilon}+\theta^{-, \varepsilon}>0 .
$$

Then we have for $t \geq 0$ :

$$
\begin{aligned}
\int_{I} \kappa_{x}^{\varepsilon} \log \kappa_{x}^{\varepsilon} & =\int_{I}\left(\theta^{+, \varepsilon}+\theta^{-, \varepsilon}\right) \log \left(\theta^{+, \varepsilon}+\theta^{-, \varepsilon}\right) \\
& \leq \int_{I} \theta^{+, \varepsilon} \log \left(\theta^{+, \varepsilon}\right)+\theta^{-, \varepsilon} \log \left(\theta^{-, \varepsilon}\right)+\theta^{+, \varepsilon} \log 2+\theta^{-, \varepsilon} \\
& \leq\left(\int_{I} \theta^{+, \varepsilon} \log \left(\theta^{+, \varepsilon}\right)+\theta^{-, \varepsilon} \log \left(\theta^{-, \varepsilon}\right)\right)+\log 2+1 \\
& \leq S(t)+2 .
\end{aligned}
$$

Here we have used Lemma 5.3 with $x=\theta^{+, \varepsilon}$ and $y=\theta^{-, \varepsilon}$ for the second line, and we have used for the third line, the fact that

$$
\int_{I} \theta^{ \pm, \varepsilon}=\frac{1}{2} \int_{I} \kappa_{x}^{\varepsilon} \pm \rho_{x}^{\varepsilon}=\frac{1}{2}\left[\kappa^{\varepsilon}(1, .)-\kappa^{\varepsilon}(-1, .)\right]=1 .
$$

Using (5.3), the result follows.

\section{Lemma 5.4 (Control of the modulus of continuity in space)}

Let $u \in C^{1}(I), u_{x}>0$, satisfying

$$
\int_{I} u_{x} \log \left(u_{x}\right) \leq c_{1}
$$

for some positive constant $c_{1}$, then we have for any $x, x+h \in I$ with $h>0$ :

$$
|u(x+h)-u(x)| \leq \frac{2\left(c_{1}+1+\log 2\right)}{|\log h|} .
$$

Proof. Let $x, x+h \in I$.

Step 1. $\left(u_{x} \in L_{\Psi}(x, x+h)\right.$ with $\Psi$ given in (3.9))

We compute

$$
\begin{aligned}
\int_{x}^{x+h} \Psi\left(u_{x}\right) & \leq \int_{I}\left(1+u_{x}\right) \log \left(1+u_{x}\right)-u_{x} \\
& \leq \int_{I} u_{x} \log \left(u_{x}\right)+\log 2 \leq c_{1}+\log 2
\end{aligned}
$$

where we have used (5.7) in the second line inequality. Hence from (3.10), we get

$$
\left\|u_{x}\right\|_{L_{\Psi}(x, x+h)} \leq c_{1}+1+\log 2 .
$$

Step 2. (Estimating the modulus of continuity)

It is easy to check that the function 1 lies in $L_{\Phi}(x, x+h)$ for $\Phi$ given by (3.9), and that $\|1\|_{L_{\Phi}(x, x+h)} \leq-\frac{1}{\log h}$. Therefore, by Hölder inequality (3.11), we obtain:

$$
\begin{aligned}
|u(x+h)-u(x)| & =\left|\int_{x}^{x+h} u_{x} \cdot 1\right| \\
& \leq 2\left\|u_{x}\right\|_{L_{\psi}(x, x+h)}\|1\|_{L_{\Phi}(x, x+h)} \leq \frac{2\left(c_{1}+1+\log 2\right)}{|\log h|}
\end{aligned}
$$


and the result follows.

Remark 5.5 As mentioned to us by Jérôme Droniou, it is possible to estimate directly the quantity $\mid u(x+$ $h)-u(x) \mid \leq \frac{A}{|\log h|}$ by splitting the integral $\int_{x}^{x+h} u_{x}$ on the set where $u_{x}$ is bigger and lower than $\lambda$, and then optimizing on the parameter $\lambda$.

\section{An interior estimate}

In this section, we give an interior estimate for the term

$$
A^{\varepsilon}=\rho_{x}^{\varepsilon}-\tau \kappa^{\varepsilon} .
$$

that will be used in the passage to the limit as $\varepsilon$ goes to zero in the regularized system. We start by deriving an equation satisfied by $A^{\varepsilon}$ :

$$
A_{t}^{\varepsilon}=(1+\varepsilon) A_{x x}^{\varepsilon}-\frac{\tau \rho_{x}^{\varepsilon}}{\kappa_{x}^{\varepsilon}} A_{x}^{\varepsilon} .
$$

We now show an interior $L^{p}$ estimate concerning the term $A^{\varepsilon}$. This estimate gives a control on the local $L^{p}$ norm of $A^{\varepsilon}$ by its global $L^{1}$ norm over $I_{T}$, and it will be used in the following section. More precisely, we have the following lemma.

Lemma 6.1 (Interior $L^{p}$ estimate). Let $0<\varepsilon<1$ and $1<p<\infty$. Then the quantity $A^{\varepsilon}$ given by (6.1) satisfies:

$$
\left\|A^{\varepsilon}\right\|_{p, V} \leq c\left(\left\|A^{\varepsilon}\right\|_{1, I_{T}}+1\right),
$$

where $V$ is an open subset of $I_{T}$ such that $V \subset \subset I_{T}$, and $c=c(p, V)>0$ is a constant independent of $\varepsilon$.

Proof. Throughout the proof, the term $c=c(p, V)>0$ is a positive constant independent of $\varepsilon$, and it may vary from line to line. A simple computation gives:

$$
\begin{aligned}
-\tau \frac{\rho_{x}^{\varepsilon}}{\kappa_{x}^{\varepsilon}} A_{x}^{\varepsilon} & =-\tau \frac{\rho_{x}^{\varepsilon}}{\kappa_{x}^{\varepsilon}}\left(\rho_{x x}^{\varepsilon}-\tau \kappa_{x}^{\varepsilon}\right) \\
& =-\tau \frac{\rho_{x}^{\varepsilon} \rho_{x x}^{\varepsilon}}{\kappa_{x}^{\varepsilon}}+\tau^{2} \rho_{x}^{\varepsilon} \\
& =-\tau\left(\kappa_{t}^{\varepsilon}-\varepsilon \kappa_{x x}^{\varepsilon}\right) .
\end{aligned}
$$

Define $\bar{\kappa}^{\varepsilon}$ as the unique solution of

$$
\begin{cases}\bar{\kappa}_{t}^{\varepsilon}=(1+\varepsilon) \bar{\kappa}_{x x}^{\varepsilon}+\kappa^{\varepsilon} & \text { on } \quad I_{T}, \\ \bar{\kappa}^{\varepsilon}=0 & \text { on } \quad \partial^{p} I_{T},\end{cases}
$$

where the existence and uniqueness of this equation is a direct consequence of the $L^{p}$ theory for parabolic equations (see for instance [18, Theorem 9.1]) using in particular the fact that $\kappa^{\varepsilon} \in C^{1}\left(\overline{I_{T}}\right)$. Moreover, from the regularity (3.4) of $\kappa^{\varepsilon}$, we can deduce that $\bar{\kappa}^{\varepsilon} \in C^{\infty}\left(I_{T}\right)$. Let

$$
\bar{A}^{\varepsilon}=-\tau\left(\bar{\kappa}_{t}^{\varepsilon}-\varepsilon \bar{\kappa}_{x x}^{\varepsilon}\right), \quad \text { with } \quad a^{\varepsilon}=A^{\varepsilon}-\bar{A}^{\varepsilon} .
$$

We calculate:

$$
\begin{aligned}
\bar{A}_{t}^{\varepsilon} & =-\tau\left[\bar{\kappa}_{t t}^{\varepsilon}-\varepsilon \bar{\kappa}_{x x t}^{\varepsilon}\right] \\
& =-\tau\left[(1+\varepsilon) \bar{\kappa}_{x x t}^{\varepsilon}+\kappa_{t}^{\varepsilon}-\varepsilon\left((1+\varepsilon) \bar{\kappa}_{x x x x}^{\varepsilon}+\kappa_{x x}^{\varepsilon}\right)\right] \\
& =-\tau(1+\varepsilon)\left(\bar{\kappa}_{x x t}^{\varepsilon}-\varepsilon \bar{\kappa}_{x x x x}^{\varepsilon}\right)-\tau\left(\kappa_{t}^{\varepsilon}-\varepsilon \kappa_{x x}^{\varepsilon}\right) \\
& =(1+\varepsilon) \bar{A}_{x x}-\frac{\tau \rho_{x}^{\varepsilon}}{\kappa_{x}^{\varepsilon}} A_{x}^{\varepsilon},
\end{aligned}
$$


where for the first two line, we have used (6.5), and for the last line, we have used (6.4). In this case, we obtain:

$$
\begin{aligned}
a_{t}^{\varepsilon} & =A_{t}^{\varepsilon}-\bar{A}_{t}^{\varepsilon} \\
& =(1+\varepsilon) A_{x x}^{\varepsilon}-\frac{\tau \rho_{x}^{\varepsilon}}{\kappa_{x}^{\varepsilon}} A_{x}^{\varepsilon}-(1+\varepsilon) \bar{A}_{x x}+\frac{\tau \rho_{x}^{\varepsilon}}{\kappa_{x}^{\varepsilon}} A_{x}^{\varepsilon} \\
& =(1+\varepsilon)\left(A_{x x}^{\varepsilon}-\bar{A}_{x x}^{\varepsilon}\right)=(1+\varepsilon) a_{x x}^{\varepsilon},
\end{aligned}
$$

where for the first line, we have used the equation (6.2). We apply Lemma 3.5 to the function $a^{\varepsilon}$, after doing parabolic rescaling of the form $\tilde{a}^{\varepsilon}(x, t)=a^{\varepsilon}\left(x, \frac{t}{1+\varepsilon}\right)$, we get:

$$
\left\|a^{\varepsilon}\right\|_{p, V} \leq c(1+\varepsilon)^{1-\frac{1}{p}}\left\|a^{\varepsilon}\right\|_{1, I_{T}},
$$

and since $0<\varepsilon<1$, we finally obtain

$$
\left\|a^{\varepsilon}\right\|_{p, V} \leq c\left\|a^{\varepsilon}\right\|_{1, I_{T}} .
$$

From the definition of $a^{\varepsilon}$, and the last inequality, we finally deduce that:

$$
\left\|A^{\varepsilon}\right\|_{p, V} \leq c\left(\left\|A^{\varepsilon}\right\|_{1, I_{T}}+\left\|\bar{A}^{\varepsilon}\right\|_{p, I_{T}}\right) .
$$

In order to complete the proof, we need to control the term $\left\|\bar{A}^{\varepsilon}\right\|_{p, I_{T}}$ in (6.6). We use the equation (6.5) satisfied by $\bar{\kappa}^{\varepsilon}$ to obtain:

$$
\begin{aligned}
\left\|\bar{A}^{\varepsilon}\right\|_{p, I_{T}} & =\tau\left\|\bar{\kappa}_{t}^{\varepsilon}-\varepsilon \bar{\kappa}_{x x}^{\varepsilon}\right\|_{p, I_{T}} \\
& =\tau\left\|\bar{\kappa}_{x x}^{\varepsilon}+\kappa^{\varepsilon}\right\|_{p, I_{T}} \\
& \leq c\left(\left\|\bar{\kappa}_{x x}^{\varepsilon}\right\|_{p, I_{T}}+\left\|\kappa^{\varepsilon}\right\|_{p, I_{T}}\right) .
\end{aligned}
$$

The $L^{p}$ estimates for parabolic equations (see [16, Lemma 2.7]) applied to (6.5) gives:

$$
\left\|\bar{\kappa}_{x x}^{\varepsilon}\right\|_{p, I_{T}} \leq \frac{c}{1+\varepsilon}\left\|\kappa^{\varepsilon}\right\|_{p, I_{T}},
$$

then (6.7), together with the fact that $0 \leq \kappa^{\varepsilon} \leq 1$ (see Remark 3.3), implies that:

$$
\left\|\bar{A}^{\varepsilon}\right\|_{p, I_{T}} \leq c\left\|\kappa^{\varepsilon}\right\|_{p, I_{T}} \leq c T^{1 / p},
$$

hence the result follows.

\section{Proof of the main theorem}

At this stage, we are ready to present the proof of our main result (Theorem 1.1). This depends essentially on the passage to the limit in the family of solutions $\left(\rho^{\varepsilon}, \kappa^{\varepsilon}\right)$ of system (2.2). Since $\kappa_{x}^{\varepsilon} \neq 0$, we multiply the first equation of (2.2) by $\kappa_{x}^{\varepsilon}$ and we rewrite system (2.2) in terms of $A^{\varepsilon}$, we obtain:

$$
\begin{cases}\kappa_{t}^{\varepsilon} \kappa_{x}^{\varepsilon}=\varepsilon \kappa_{x}^{\varepsilon} \kappa_{x x}^{\varepsilon}+\rho_{x}^{\varepsilon} A_{x}^{\varepsilon} & \text { on } I_{T} \\ \rho_{t}^{\varepsilon}=\varepsilon \rho_{x x}^{\varepsilon}+A_{x}^{\varepsilon} & \text { on } I_{T} .\end{cases}
$$

We will pass to the limit in the framework of viscosity solutions for the first equation of (7.1), and in the distributional sense for the second equation. We start with the following proposition.

\section{Proposition 7.1 (Local uniform convergence)}

The sequences $\left(\rho^{\varepsilon}\right)_{\varepsilon},\left(\rho_{x}^{\varepsilon}\right)_{\varepsilon},\left(\kappa^{\varepsilon}\right)_{\varepsilon},\left(A^{\varepsilon}\right)_{\varepsilon}$ and $\left(A_{x}^{\varepsilon}\right)_{\varepsilon}$ converge (up to extraction of a subsequence) locally uniformly in $I_{T}$ as $\varepsilon$ goes to zero. 
Proof. Let $V$ be an open compactly contained subset of $I_{T}$. The constants that will appear in the proof are all independent of $\varepsilon$. However, they may depend on other fixed parameters including $V$. The idea is to give an $\varepsilon$-uniform control of the modulus of continuity in space and in time of the quantities mentioned in Proposition (7.1), which gives the local uniform convergence. The $\varepsilon$-uniform control on the space modulus of continuity will be derived from the Corollary 5.2 and Lemma 5.4, while the $\varepsilon$-uniform control on the time modulus of continuity will be derived from Lemma 3.4. The proof is divided into five steps.

Step 1. (Convergence of $A^{\varepsilon}$ and $A_{x}^{\varepsilon}$ )

From (3.5), we know that $\left\|\frac{\rho_{x}^{\varepsilon}}{\kappa_{x}^{\varepsilon}}\right\|_{\infty} \leq 1$. We apply the interior $L^{p}, p>1$, estimates for parabolic equations (see for instance [19, Theorem 7.13, page 172]) to the term $A^{\varepsilon}$ satisfying (6.2), we obtain:

$$
\left\|A^{\varepsilon}\right\|_{W_{p}^{2,1}(V)} \leq c_{2}\left\|A^{\varepsilon}\right\|_{p, V^{\prime}},
$$

where $V^{\prime}$ is any open subset of $I_{T}$ satisfying $V \subset \subset V^{\prime} \subset \subset I_{T}$. The constant $c_{2}=c_{2}\left(p, \tau, V, V^{\prime}\right)$ can be chosen independent of $\varepsilon$ first by applying a parabolic rescaling of (6.2), and then using the fact that the factor multiplied by $A_{x x}^{\varepsilon}$ in (6.2) satisfying $1 \leq 1+\varepsilon \leq 2$. At this point, we apply Lemma 6.1 for $A^{\varepsilon}$ on $V^{\prime}$, we get:

$$
\left\|A^{\varepsilon}\right\|_{p, V^{\prime}} \leq c_{3}\left(\left\|A^{\varepsilon}\right\|_{1, I_{T}}+1\right)
$$

and hence the above two equations (7.2) and (7.3) give:

$$
\left\|A^{\varepsilon}\right\|_{W_{p}^{2,1}(V)} \leq c_{4}\left(\left\|A^{\varepsilon}\right\|_{1, I_{T}}+1\right) .
$$

We estimate the right hand side of (7.4) in the following way:

$$
\begin{aligned}
\left\|A^{\varepsilon}\right\|_{1, I_{T}} & =\int_{I_{T}}\left|\rho_{x}^{\varepsilon}-\tau \kappa^{\varepsilon}\right| \\
& \leq \int_{I_{T}} \kappa_{x}^{\varepsilon}+\tau\left|\kappa^{\varepsilon}\right| \\
& \leq(2+\tau) T,
\end{aligned}
$$

where we have used the fact that $\left|\rho_{x}^{\varepsilon}\right|<\kappa_{x}^{\varepsilon}$ (see (3.5) of Theorem 3.1) in the second line, and the fact that $0 \leq\left|\kappa^{\varepsilon}\right| \leq 1$ (see Remark 3.3) in the last line. Therefore, inequality (7.4) implies:

$$
\left\|A^{\varepsilon}\right\|_{W_{p}^{2,1}(V)} \leq c_{5}, \quad 1<p<\infty .
$$

We use the above inequality for $p>3$. In this case, the Sobolev embedding in Hölder spaces (see [16, Lemma 2.8]) gives:

$$
W_{p}^{2,1}(V) \hookrightarrow C^{1+\alpha, \frac{1+\alpha}{2}}(V), \quad \alpha=1-3 / p
$$

and hence (7.5) implies:

$$
\left\|A^{\varepsilon}\right\|_{C^{1+\alpha, \frac{1+\alpha}{2}}(V)} \leq c_{6},
$$

which guarantees the equicontinuity and the equiboundedness of $\left(A^{\varepsilon}\right)_{\varepsilon}$ and $\left(A_{x}^{\varepsilon}\right)_{\varepsilon}$. By the Arzela-Ascoli Theorem (see for instance [3]), we finally obtain

$$
A^{\varepsilon} \longrightarrow A \text { and } A_{x}^{\varepsilon} \longrightarrow A_{x}
$$

up to a subsequence, uniformly on $V$ as $\varepsilon \rightarrow 0$.

Step 2. (Convergence of $\kappa^{\varepsilon}$ ) 
We control the modulus of continuity of $\kappa^{\varepsilon}$ in space and in time, locally uniformly in $\varepsilon$.

Step 2.1. (Control of the modulus of continuity in time)

The first equation of (7.1) gives:

$$
\kappa_{t}^{\varepsilon}=\varepsilon \kappa_{x x}^{\varepsilon}+\frac{\rho_{x}^{\varepsilon}}{\kappa_{x}^{\varepsilon}} A_{x}^{\varepsilon}
$$

and hence, using the fact that $\left\|\frac{\rho_{x}^{\varepsilon}}{\kappa_{x}^{\varepsilon}}\right\|_{\infty} \leq 1$, together with (7.6), we get:

$$
\left\|\kappa_{t}^{\varepsilon}-\varepsilon \kappa_{x x}^{\varepsilon}\right\|_{\infty, V} \leq\left\|\frac{\rho_{x}^{\varepsilon}}{\kappa_{x}^{\varepsilon}}\right\|_{\infty, V}\left\|A_{x}\right\|_{\infty, V} \leq c_{6} .
$$

Also, by (3.6), we have:

$$
\left\|\kappa^{\varepsilon}\right\|_{\infty, V} \leq 1 .
$$

This uniform bound on $\kappa^{\varepsilon}$ together with (7.8) permit to use Lemma 3.4 to conclude that

$$
\left|\kappa^{\varepsilon}(x, t)-\kappa^{\varepsilon}(x, t+h)\right| \leq c_{7} h^{\beta}, \quad(x, t),(x, t+h) \in V, \quad 0<\beta<1,
$$

which controls the modulus of continuity of $\kappa^{\varepsilon}$ with respect to $t$ uniformly in $\varepsilon$. We now move to control the modulus of continuity in space.

Step 2.2 (An $\varepsilon$-uniform bound on $S(0)$ )

Recall the definition (5.2) of $S(t)$ :

$$
S(t)=\int_{I} \sum_{ \pm} \theta^{ \pm, \varepsilon}(x, t) \log \theta^{ \pm, \varepsilon}(x, t) d x
$$

with

$$
\theta^{ \pm, \varepsilon}=\frac{\kappa_{x}^{\varepsilon} \pm \rho_{x}^{\varepsilon}}{2}
$$

Hence

$$
S(0)=\int_{I} \frac{\kappa_{x}^{0, \varepsilon}+\rho_{x}^{0, \varepsilon}}{2} \log \left(\frac{\kappa_{x}^{0, \varepsilon}+\rho_{x}^{0, \varepsilon}}{2}\right)+\int_{I} \frac{\kappa_{x}^{0, \varepsilon}-\rho_{x}^{0, \varepsilon}}{2} \log \left(\frac{\kappa_{x}^{0, \varepsilon}-\rho_{x}^{0, \varepsilon}}{2}\right) .
$$

Using the elementary identities $x \log x \leq x^{2}$ and $(x \pm y)^{2} \leq 2\left(x^{2}+y^{2}\right)$, we compute:

$$
\begin{aligned}
S(0) & \leq \int_{I}\left(\frac{\kappa_{x}^{0, \varepsilon}+\rho_{x}^{0, \varepsilon}}{2}\right)^{2}+\int_{I}\left(\frac{\kappa_{x}^{0, \varepsilon}-\rho_{x}^{0, \varepsilon}}{2}\right)^{2} \\
& \leq\left\|\rho_{x}^{0, \varepsilon}\right\|_{2, I}^{2}+\left\|\kappa_{x}^{0, \varepsilon}\right\|_{2, I}^{2} .
\end{aligned}
$$

From (4.1), we know that:

$$
\left|\rho_{x}^{0, \varepsilon}\right|=\left|\frac{\rho_{x}^{0}+\varepsilon \tau \phi^{\prime}}{(1+\varepsilon)^{2}}\right| \leq \frac{\left|\rho_{x}^{0}\right|+\varepsilon}{(1+\varepsilon)^{2}} \leq\left|\rho_{x}^{0}\right|+1,
$$

and

$$
\left|\kappa_{x}^{0, \varepsilon}\right|=\left|\frac{\kappa_{x}^{0}+\varepsilon}{1+\varepsilon}\right| \leq\left|\kappa_{x}^{0}\right|+1 .
$$

Using the above two inequalities into (7.10), we deduce that:

$$
S(0) \leq 2\left(\left\|\rho_{x}^{0}\right\|_{2, I}^{2}+\left\|\kappa_{x}^{0}\right\|_{2, I}^{2}+2\right) .
$$




\section{Step 2.3. (Control of the modulus of continuity in space and conclusion)}

We use the uniform bound obtained for $S(0)$ in Step 2.1, together with the special control (5.6) of $\kappa_{x}^{\varepsilon}$ given in Corollary 5.2, we get for all $0 \leq t \leq T$ :

$$
\int_{I} \kappa_{x}^{\varepsilon}(x, t) \log \left(\kappa_{x}^{\varepsilon}(x, t)\right) d x \leq 2\left(\left\|\rho_{x}^{0}\right\|_{2, I}^{2}+\left\|\kappa_{x}^{0}\right\|_{2, I}^{2}+2\right)+\frac{\tau^{2} T}{2}+2,
$$

therefore

$$
\int_{I} \kappa_{x}^{\varepsilon}(x, t) \log \left(\kappa_{x}^{\varepsilon}(x, t)\right) d x \leq c_{8}, \quad \forall 0 \leq t \leq T .
$$

Inequality (7.11) permits to use Lemma 5.4 , hence we obtain:

$$
\left|\kappa^{\varepsilon}(x+h, t)-\kappa^{\varepsilon}(x, t)\right| \leq \frac{c_{9}}{|\log h|}, \quad(x, t),(x+h, t) \in I_{T},
$$

Inequalities (7.9) and (7.12) give the equicontinuity of the sequence $\left(\kappa^{\varepsilon}\right)_{\varepsilon}$ on $V$, and again by the ArzelaAscoli Theorem, we get:

$$
\kappa^{\varepsilon} \rightarrow \kappa
$$

up to a subsequence, uniformly on $V$ as $\varepsilon \rightarrow 0$.

Step 3. (Convergence of $\rho^{\varepsilon}$ )

As in step 2, we control the modulus of continuity of $\rho^{\varepsilon}$ in space and in time, locally uniformly with respect to $\varepsilon$.

\section{Step 3.1. (Control of the modulus of continuity in time)}

The second equation of (7.1) gives:

$$
\rho_{t}^{\varepsilon}-\varepsilon \rho_{x x}^{\varepsilon}=A_{x}^{\varepsilon}
$$

hence, from (7.6), we deduce that:

$$
\left\|\rho_{t}^{\varepsilon}-\varepsilon \rho_{x x}^{\varepsilon}\right\|_{\infty, V} \leq c_{6}
$$

and from (3.6), we have:

$$
\left\|\rho^{\varepsilon}\right\|_{\infty, V} \leq 1
$$

The above two inequalities permit to use Lemma 3.4, we finally get:

$$
\left|\rho^{\varepsilon}(x, t)-\rho^{\varepsilon}(x, t+h)\right| \leq c_{7} h^{\beta}, \quad(x, t),(x, t+h) \in V, \quad 0<\beta<1,
$$

which controls the modulus of continuity of $\rho^{\varepsilon}$ with respect to $t$ uniformly in $\varepsilon$.

Step 3.2. (Control of the modulus of continuity in space and conclusion)

The control of the space modulus of continuity is based on the following observation. From (3.5), we know that $\left|\rho_{x}^{\varepsilon}\right| \leq \kappa_{x}^{\varepsilon}$ on $I_{T}$. Using this inequality, we get, for every $(x, t),(x+h, t) \in I_{T}$ :

$$
\left|\rho^{\varepsilon}(x+h, t)-\rho^{\varepsilon}(x, t)\right| \leq \int_{x}^{x+h}\left|\rho_{x}^{\varepsilon}(y, t)\right| d y \leq \int_{x}^{x+h} \kappa_{x}^{\varepsilon}(y, t) d y \leq\left|\kappa^{\varepsilon}(x+h, t)-\kappa^{\varepsilon}(x, t)\right| .
$$

Inequality (7.12) gives immediately that:

$$
\left|\rho^{\varepsilon}(x+h, t)-\rho^{\varepsilon}(x, t)\right| \leq \frac{c_{9}}{|\log h|}, \quad(x, t),(x+h, t) \in I_{T} .
$$


From (7.14) and (7.15), we deduce that:

$$
\rho^{\varepsilon} \rightarrow \rho
$$

up to a subsequence, uniformly on $V$ as $\varepsilon \rightarrow 0$.

Step 4. (Convergence of $\rho_{x}^{\varepsilon}$ and conclusion)

In fact, this follows from Step 1, Step 2, and the fact that

$$
\rho_{x}^{\varepsilon}=A^{\varepsilon}+\tau \kappa^{\varepsilon} \rightarrow \rho_{x}
$$

uniformly on $V$ as $\varepsilon \rightarrow 0$. In this case, we also deduce that

$$
A=\rho_{x}-\tau \kappa .
$$

The proof of Proposition 7.1 is done.

We now move to the proof of the main result.

Proof of Theorem 1.1. We first remark that $\kappa^{\varepsilon}$ is a viscosity solution of the first equation of (7.1):

$$
\kappa_{t}^{\varepsilon} \kappa_{x}^{\varepsilon}-\varepsilon \kappa_{x}^{\varepsilon} \kappa_{x x}^{\varepsilon}-\rho_{x}^{\varepsilon} A_{x}^{\varepsilon}=0 \quad \text { on } \quad I_{T} .
$$

Remark 7.2 The equation (7.18) can be viewed as the following Hamilton-Jacobi equation of second order:

$$
H^{\varepsilon}\left(X, D \kappa^{\varepsilon}, D^{2} \kappa^{\varepsilon}\right)=0, \quad X=(x, t) \in I_{T}
$$

with

$$
D \kappa^{\varepsilon}=\left(\kappa_{x}^{\varepsilon}, \kappa_{t}^{\varepsilon}\right) \quad \text { and } \quad D^{2} \kappa^{\varepsilon}=\left(\begin{array}{cc}
\kappa_{x x}^{\varepsilon} & \kappa_{x t}^{\varepsilon} \\
\kappa_{t x}^{\varepsilon} & \kappa_{t t}^{\varepsilon}
\end{array}\right),
$$

where $H^{\varepsilon}$ is the Hamiltonian function given by:

$$
\begin{aligned}
H^{\varepsilon}: I_{T} \times \mathbb{R}^{2} \times M_{\text {sym }}^{2 \times 2} & \longrightarrow \mathbb{R} \\
(X, p, M) & \longmapsto H^{\varepsilon}(X, p, M)=p_{1} p_{2}-\varepsilon p_{1} M_{11}-\rho_{x}^{\varepsilon}(X) A_{x}^{\varepsilon}(X),
\end{aligned}
$$

$p=\left(p_{1}, p_{2}\right)$ and $M=\left(M_{i j}\right)_{i, j=1,2}$.

From (7.7) and (7.17), we deduce that $\left(H^{\varepsilon}\right)_{\varepsilon}$ converges locally uniformly in $I_{T} \times \mathbb{R}^{2} \times M_{s y m}^{2 \times 2}$ to the function $H$ given by:

$$
\begin{aligned}
H: I_{T} \times \mathbb{R}^{2} \times M_{s y m}^{2 \times 2} & \longrightarrow \mathbb{R} \\
(X, p, M) & \longmapsto H(X, p, M)=p_{1} p_{2}-\rho_{x}(X) A_{x}(X) .
\end{aligned}
$$

This, together with the local uniform convergence of $\kappa^{\varepsilon}$ to $\kappa$ (see 7.13), and the fact that $\kappa^{\varepsilon}$ is a viscosity solution of (7.18), permit to use the stability of viscosity solutions (see Theorem 3.7), which proves that $\kappa$ is a viscosity solution of

$$
H\left(X, D \kappa, D^{2} \kappa\right)=\kappa_{t} \kappa_{x}-\rho_{x} A_{x}=0 \quad \text { in } \quad I_{T} .
$$

We now pass to the limit $\varepsilon \rightarrow 0$ in the second equation of (7.1), we obtain

$$
\rho_{t}=A_{x} \in C\left(I_{T}\right) \quad \text { in } \quad \mathcal{D}^{\prime}\left(I_{T}\right) .
$$

From (7.22) and (7.23), we get:

1. $\kappa$ is a viscosity solution of $\kappa_{t} \kappa_{x}=\rho_{t} \rho_{x}$ in $I_{T}$;

2. $\rho$ is a distributional solution of $\rho_{t}=\rho_{x x}-\tau \kappa_{x}$ in $I_{T}$. 
Inequality (1.12) can be easily obtained by testing against a nonnegative function $\phi \in C_{0}^{\infty}\left(I_{T}\right)$. Finally, let us show how to retrieve the initial and boundary conditions. Indeed, the local uniform convergence $\left(\rho^{\varepsilon}, \kappa^{\varepsilon}\right) \rightarrow(\rho, \kappa)$, together with the uniform control of the modulus of continuity of these solutions:

- with respect to $x$ near $\partial I \times[0, T]$ by $(7.12)$;

- with respect to $t$ near $I \times\{t=0\}$, away from 0 and 1 by (7.9),

and the fact that $\kappa^{0, \varepsilon} \rightarrow \kappa^{0}, \rho^{0, \varepsilon} \rightarrow \rho^{0}$ uniformly in $\bar{I}$, show that $(\rho, \kappa) \in\left(C\left(\overline{I_{T}}\right)\right)^{2}$, so the initial and boundary conditions are satisfied pointwisely, and the proof of the main result is done.

\section{Application: simulations for the evolution of elastoviscoplatic materials}

Motivated by the simulation of the elastoviscoplastic behavior that are formulated by the model of Groma, Csikor and Zaiser [12], this section is devoted to write down the equations of the displacement vector $u$ inside the crystal when a constant exterior shear stress $\tau$ (in this section we take $\tau>0$ ) is applied on the boundary walls (see Figure 1). Also, at the end of this section, we present some numerical simulations revealing the evolution of a crystal. Here, as we have already mentioned in the introduction, we suppose that the distribution of dislocations is invariant by translation in the $y$-direction. We consider a 2-dimensional crystal (Figure 1) with the displacement vector:

$$
u=\left(u_{1}, u_{2}\right): \mathbb{R}^{2} \longmapsto \mathbb{R}^{2} .
$$

We rename the coordinates $(x, y)$ as $\left(x_{1}, x_{2}\right)$, and we call $\left(e_{1}, e_{2}\right)$ the corresponding orthonormal basis. We define the total strain by:

$$
\varepsilon_{i j}(u)=\frac{1}{2}\left(\partial_{j} u_{i}+\partial_{i} u_{j}\right) \quad \text { with } \quad \partial_{j} u_{i}=\frac{\partial u_{i}}{\partial x_{j}}, \quad i, j=1,2 .
$$

This total strain could be decomposed into two parts as follows:

$$
\varepsilon_{i j}(u)=\varepsilon_{i j}^{e}(u)+\varepsilon_{i j}^{p},
$$

where $\varepsilon^{e}(u)$ is the elastic strain and $\varepsilon^{p}$ is the plastic strain which is given by:

$$
\varepsilon_{i j}^{p}=\rho \varepsilon_{i j}^{0},
$$

with

$$
\varepsilon_{i j}^{0}=\frac{1}{2}\left(1-\delta_{i j}\right),
$$

in the special case of a single slip system where dislocations move following the Burgers vector $\vec{b}=e_{1}\left(\delta_{i j}\right.$ is the usual Kronecker symbol). Here $\rho$ is the resolved plastic strain. The stress field $\sigma$ inside the crystal is given by:

$$
\sigma_{i j}=2 \mu \varepsilon_{i j}^{e}(u)+\lambda \delta_{i j}\left(\sum_{k=1,2} \varepsilon_{k k}^{e}(u)\right),
$$

with $\lambda, \mu>0$ are the constants of Lamé coefficients of the crystal that are assumed (for simplification) to be isotropic. This stress field $\sigma$ has to satisfy the equation of elasticity:

$$
\sum_{j=1,2} \frac{\partial \sigma_{i j}}{\partial x_{j}}=0 .
$$


Finally, the functions $\rho, \kappa$ (solutions of (1.3)) and $u$ are solutions of the following coupled system:

$$
\left\{\begin{array}{lll}
\sum_{j=1,2} \frac{\partial \sigma_{i j}}{\partial x_{j}} & =0 & \text { on } I \times(0, \infty), \\
\sigma_{i j} & =2 \mu \varepsilon_{i j}^{e}(u)+\lambda \delta_{i j}\left(\sum_{k=1,2} \varepsilon_{k k}^{e}(u)\right) & \text { on } I \times(0, \infty), \\
\varepsilon_{i j}^{e} & =\frac{1}{2}\left(\partial_{j} u_{i}+\partial_{i} u_{j}\right)-\rho \varepsilon_{i j}^{0} & \text { on } I \times(0, \infty), \\
\kappa_{t} \kappa_{x} & =\rho_{t} \rho_{x} & \text { on } I \times(0, \infty), \\
\rho_{t} & =\rho_{x x}-\tau \kappa_{x} & \text { on } I \times(0, \infty),
\end{array}\right.
$$

Equation (8.4) can be reformulated as:

$$
\left\{\begin{array}{l}
(\lambda+2 \mu) \Delta u_{1}+(\lambda+\mu) \partial_{12} u_{2}=0 \\
(\lambda+2 \mu) \Delta u_{2}+(\lambda+\mu) \partial_{21} u_{1}=\mu \partial_{1} \rho .
\end{array}\right.
$$

Here $\partial_{2} \rho=0$ is due to the homogeneity of the distribution of dislocations in the $e_{2}$-direction.

Calculation of $u$. We first calculate the value of the displacement $u$ on the boundary walls. Remark first that since we are applying a constant shear stress field on the walls, the stress field $\sigma$ there can be evaluated as: $\sigma \cdot n= \pm \tau e_{2}, n= \pm e_{1}$, for $x= \pm 1$,

$$
\sigma=\left(\begin{array}{ll}
0 & \tau \\
\tau & 0
\end{array}\right), \quad \text { on } \quad \partial I
$$

Using (8.7) and (8.3), we can derive the following equations on the boundary:

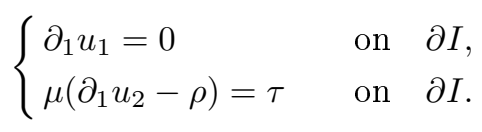

Equation (8.6) leads to the following two equations inside $I$ :

$$
\begin{cases}\partial_{1}\left[(\lambda+2 \mu) \partial_{1} u_{1}\right]=0 & \text { on } \quad I \\ \partial_{1}\left(\partial_{1} u_{2}-\rho\right)=0 & \text { on } \quad I .\end{cases}
$$

Combining (8.8) and (8.9), with

$$
u_{1}\left(0, x_{2}\right)=u_{2}\left(0, x_{2}\right)=0
$$

finally lead to:

$$
\begin{cases}u_{1}\left(x_{1}, x_{2}\right)=0, & \left(x_{1}, x_{2}\right) \in I \times \mathbb{R} \\ u_{2}\left(x_{1}, x_{2}\right)=\frac{\tau}{\mu} x_{1}+\int_{0}^{x_{1}} \rho(x) d x, & \left(x_{1}, x_{2}\right) \in I \times \mathbb{R} .\end{cases}
$$

Formal computation of the long time solution of system (1.3). All computations that will be done here are formal. We seek to calculate long time solutions for system (1.3). For this reason, we first calculate the long time solution (stationary solution, $\kappa_{t}^{\varepsilon}=\rho_{t}^{\varepsilon}=0$ ) of the regularized $\varepsilon$-system $(2.2)$, and then we pass to the limit $\varepsilon \rightarrow 0$. Doing some computations, the long time $(t \rightarrow \infty)$ solution for the $\varepsilon$-system is given by:

$$
\rho^{\varepsilon}(x, t)=B\left(\cosh \left(\frac{\tau x}{1+\varepsilon}\right)-\cosh \left(\frac{\tau}{1+\varepsilon}\right)\right) \quad \text { and } \quad \kappa^{\varepsilon}(x, t)=B \sinh \left(\frac{\tau}{1+\varepsilon}\right),
$$


with $B=1 / \sinh \left(\frac{\tau}{1+\varepsilon}\right)$. Passing (again formally) to the limit as $\varepsilon \rightarrow 0$ in (8.11), and using (8.10), we can compute the long time displacement $u_{2}$ inside the material. In fact, we have:

$$
u_{2}\left(x_{1}, x_{2}\right)=\left(\frac{\tau}{\mu}-\frac{\cosh \tau}{\sinh \tau}\right) x_{1}+\frac{\sinh \tau x_{1}}{\tau \sinh \tau} .
$$

Numerical simulations. The displacement $u$ is numerically computed by discretizing system (1.3) using an upwind numerical scheme. The space and time steps in this scheme are well chosen in order to satisfy a CFL condition (for the details, see [14, Appendix]). In Figure 2, we show successively the initial state of the crystal at time $t=0$ without any applied stress, then the instantaneous (elastic) deformation of the crystal when we apply the shear stress $\tau>0$ at time $t=0^{+}$. The deformation of the crystal evolves in time and finally converges numerically to some particular deformation which is shown on the last picture after a very long time. This kind of behavior is called elasto-visco-plasticity in mechanics. Moreover, on the last picture, we observe the presence of boundary layer deformations. This effect is directly related to the introduction of the back stress $\tau_{b}=\frac{\theta_{x}^{+}-\theta_{x}^{-}}{\theta^{+}+\theta^{-}}$in the model.

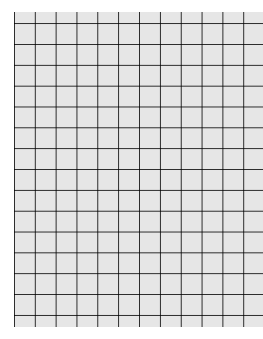

a) $t=0^{-}$

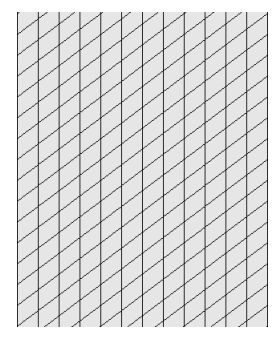

b) $t=0^{+}$

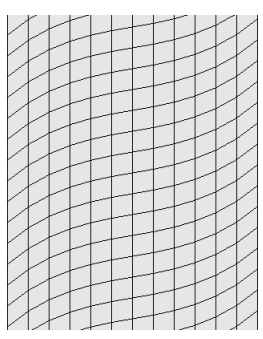

c) $t=+\infty$

Figure 2: Deformation of a slab for model (1.3).

Remark 8.1 In the last image of Figure 2, it seems that the long time displacement $u_{2}$ inside the material is coherent with our formal computation for the term $u_{2}$ (see equation (8.12)).

\section{Appendix}

\section{A1. Proof of Lemma 3.4 (control of the modulus of continuity in time)}

Let $V$ be a compactly contained subset of $I_{T}$. Throughout the proof, the constant $c$ may take several values but only depending on $V$. Since $V \subset \subset I_{T}$, then there is a rectangular cube of the form

$$
\mathcal{Q}=\left(x_{1}, x_{2}\right) \times\left(t_{1}, t_{2}\right),
$$

such that $V \subset \subset \mathcal{Q} \subset \subset I_{T}$. In this case, there exists a constant $\varepsilon_{0}$, also depending on $V$ such that for any

$$
0<\varepsilon<\varepsilon_{0}
$$

and any $(x, t) \in V$, we have:

$$
(x-2 \sqrt{\varepsilon}, x+2 \sqrt{\varepsilon}) \times\{t\} \subset \mathcal{Q} .
$$

Moreover, for any $(x, t),(x, t+h) \in V$, we can always find two intervals $\mathcal{I}$ and $\mathcal{J}$ such that

$$
(t, t+h) \subset \mathcal{I} \subset \subset \mathcal{J} \text {, with }\{x\} \times \mathcal{I} \subset \mathcal{Q} \text { and }\{x\} \times \mathcal{J} \subset \mathcal{Q} .
$$


Let us indicate that these intervals might have different lengths depending on $h$ and $V$ but we always have

$$
|\mathcal{J}|,|\mathcal{I}| \leq\left|t_{2}-t_{1}\right|
$$

Consider the following rescaling of the function $u^{\varepsilon}$ defined by:

$$
\tilde{u}^{\varepsilon}(x, t)=u^{\varepsilon}(\sqrt{\varepsilon} x, t) .
$$

This function satisfies

$$
\tilde{u}_{t}^{\varepsilon}=\tilde{u}_{x x}^{\varepsilon}+\tilde{f}^{\varepsilon}, \quad(x, t) \in(0,1 / \sqrt{\varepsilon}) \times(0, T),
$$

where $\tilde{f}^{\varepsilon}(x, t)=f^{\varepsilon}(\sqrt{\varepsilon} x, t)$. Take $\left(x_{0}, t_{0}\right),\left(x_{0}, t_{0}+h\right)$ in $V$, and let

$$
\mathcal{Q}_{1}=\left(x_{0}-\sqrt{\varepsilon}, x_{0}+\sqrt{\varepsilon}\right) \times \mathcal{I} \quad \text { and } \quad \mathcal{Q}_{2}=\left(x_{0}-2 \sqrt{\varepsilon}, x_{0}+2 \sqrt{\varepsilon}\right) \times \mathcal{J} .
$$

These two cylinders are transformed by the above rescaling into

$$
\tilde{\mathcal{Q}}_{1}=\left(\frac{x_{0}}{\sqrt{\varepsilon}}-1, \frac{x_{0}}{\sqrt{\varepsilon}}+1\right) \times \mathcal{I} \quad \text { and } \quad \tilde{\mathcal{Q}}_{2}=\left(\frac{x_{0}}{\sqrt{\varepsilon}}-2, \frac{x_{0}}{\sqrt{\varepsilon}}+2\right) \times \mathcal{J} .
$$

We apply the interior $L^{p}, p>3$, estimates for parabolic equations (see for instance [19, Theorem 7.13, page $172])$ to the function $\tilde{u}^{\varepsilon}$ over the domains $\tilde{\mathcal{Q}}_{1} \subset \subset \tilde{\mathcal{Q}}_{2}$, we get

$$
\left\|\tilde{u}^{\varepsilon}\right\|_{W_{p}^{2,1}\left(\tilde{\mathcal{Q}}_{1}\right)} \leq c\left(\left\|\tilde{u}^{\varepsilon}\right\|_{p, \tilde{\mathcal{Q}}_{2}}+\left\|\tilde{f}^{\varepsilon}\right\|_{p, \tilde{\mathcal{Q}}_{2}}\right) .
$$

Using the local $\varepsilon$-uniform boundedness of $\left(u^{\varepsilon}\right)_{\varepsilon}$, and $\left(f^{\varepsilon}\right)_{\varepsilon}$, we get:

$$
\left\|\tilde{u}^{\varepsilon}\right\|_{L^{p}\left(\tilde{\mathcal{Q}}_{2}\right)}^{p} \leq c \text { and }\left\|\tilde{f}^{\varepsilon}\right\|_{L^{p}\left(\tilde{\mathcal{Q}}_{2}\right)}^{p} \leq c .
$$

hence, inequality (9.2) implies:

$$
\left\|\tilde{u}^{\varepsilon}\right\|_{W_{p}^{2,1}\left(\tilde{\mathcal{Q}}_{1}\right)} \leq c .
$$

We use the Sobolev embedding in Hölder spaces (see for instance [16, Lemma 2.8]):

$$
W_{p}^{2,1}\left(\tilde{\mathcal{Q}}_{1}\right) \hookrightarrow C^{1+\alpha \frac{1+\alpha}{2}}\left(\tilde{\mathcal{Q}}_{1}\right), \quad p>3, \alpha=1-3 / p,
$$

to obtain, from (9.3), that:

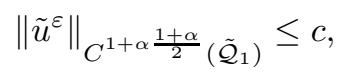

and hence

$$
\frac{\left|\tilde{u}^{\varepsilon}\left(x_{0} / \sqrt{\varepsilon}, t_{0}+h\right)-\tilde{u}^{\varepsilon}\left(x_{0} / \sqrt{\varepsilon}, t_{0}\right)\right|}{h^{\frac{1+\alpha}{2}}} \leq c
$$

then from (9.1),

$$
\frac{\left|u^{\varepsilon}\left(x_{0}, t_{0}+h\right)-u^{\varepsilon}\left(x_{0}, t_{0}\right)\right|}{h^{\frac{1+\alpha}{2}}} \leq c .
$$

Choosing $\beta=\frac{1+\alpha}{2}$ we get the desired result.

\section{A2. Proof of Lemma 3.5 (An interior estimate for the heat equation)}

Recall that $a$ is a solution of the heat equation on $I_{T}$,

$$
a_{t}=a_{x x} .
$$


The proof of lemma 3.5 is a direct computation using a mean value formula for solutions of the heat equations. Usually, basic mean value formulae of the solution of the heat equation are expressed through unbounded kernels (see for example [7, Theorem 1]), where $a$ can be expressed as:

$$
a\left(x_{0}, t_{0}\right)=\left(4 \pi r^{2}\right)^{-1 / 2} \int_{\Omega_{r}\left(x_{0}, t_{0}\right)} a(x, t) \frac{\left(x_{0}-x\right)^{2}}{4\left(t_{0}-t\right)^{2}} d x d t .
$$

Here, $\left(x_{0}, t_{0}\right) \in I_{T},(x, t) \in \Omega_{r}\left(X_{0}\right)$, and $r>0$ small enough in order to ensure that the parabolic ball of radius $r$ :

$$
\Omega_{r}\left(x_{0}, t_{0}\right)=\left\{(x, t) ; t_{0}-r^{2}<t<t_{0}, \quad\left(x-x_{0}\right)^{2}<2\left(t_{0}-t\right) \log \left(\frac{r^{2}}{t_{0}-t}\right)\right\} \subset I_{T} .
$$

In our case, we need a mean value formula similar to (9.4) but with a bounded kernel on $\Omega_{r}\left(x_{0}, t_{0}\right)$. In [10], the authors have given such a representation formula for the solution of the heat equation. The following is a direct corollary of [10, Theorem 3.1]):

Corollary 9.1 (Mean value formula with bounded kernels, [10, Theorem 3.1]) Let $u \in C^{2}(\mathcal{D})$ be a solution of the heat equation:

$$
u_{t}=u_{x x} \quad \text { on } \quad \mathcal{D},
$$

where $\mathcal{D}$ is an open subset of $\mathbb{R}^{2}$ containing the modified unit parabolic ball $\Omega_{r}^{\prime}\left(x_{0}, t_{0}\right), r>0$, with

$$
\Omega_{r}^{\prime}\left(x_{0}, t_{0}\right)=\left\{(x, t) ; t_{0}-r^{2}<t<t_{0},\left|x-x_{0}\right|^{2}<8\left(t_{0}-t\right) \log \left(\frac{r^{2}}{t_{0}-t}\right)\right\} .
$$

Then we have:

$$
u\left(x_{0}, t_{0}\right)=\frac{\bar{c}}{\left|\Omega_{r}^{\prime}\left(x_{0}, t_{0}\right)\right|} \int_{\Omega_{r}^{\prime}\left(x_{0}, t_{0}\right)} u(x, t) E\left(\frac{x-x_{0}}{r}, \frac{t-t_{0}}{r^{2}}\right) d x d t,
$$

where $\bar{c}>0,\left|\Omega_{r}^{\prime}\left(x_{0}, t_{0}\right)\right|=\bar{c} r^{3}$, and the kernel $E$ satisfies:

$$
\|E(x, t)\|_{\infty, \Omega_{1}^{\prime}(0,0)} \leq c,
$$

and $c>0$ is a fixed positive constant.

Remark 9.2 In the above corollary, which is an application of [10, Theorem 3.1] in the case $m=3$, an explicit expression of $E$ can be given by:

$$
E(x, t)=\frac{\omega_{3}}{16 \pi^{2}}\left(-x^{2}+8 t \log (-t)\right)^{3 / 2}\left[\frac{x^{2}}{4 t^{2}}+\frac{3\left(-x^{2}+8 t \log (-t)\right)}{20 t^{2}}\right],
$$

where $\omega_{3}$ is the volume of the unit ball in $\mathbb{R}^{3}$. For a more general expression of $E$, we send the reader to [10, Equality (3.6) of Theorem 3.1].

Acknowledgments. This work was supported by the contract ANR MICA (2006-2009). The authors would like to thank Jérôme Droniou for his valuable remarks while reading the manuscript of the paper.

\section{References}

[1] R. A. Adams, Sobolev spaces, Academic Press [A subsidiary of Harcourt Brace Jovanovich, Publishers], New York-London, 1975. Pure and Applied Mathematics, Vol. 65.

[2] G. Barles, Solutions de viscosité des équations de Hamilton-Jacobi, vol. 17 of Mathématiques \& Applications (Berlin) [Mathematics \& Applications], Springer-Verlag, Paris, 1994. 
[3] H. Brezis, Analyse fonctionnelle, Collection Mathématiques Appliquées pour la Maîtrise. [Collection of Applied Mathematics for the Master's Degree], Masson, Paris, 1983. Théorie et applications. [Theory and applications].

[4] M. Cannone, A. El hajJ, R. Monneau, and F. Ribaud, Global existence of a system of non-linear transport equations describing the dynamics of dislocation densities, preprint.

[5] A. El HAJJ, Well-posedness theory for a non-conservative burgers type system arising in dislocation dynamics, SIAM J. Math. Anal., 39 (2007), pp. 965-986.

[6] A. El Haju And N. ForCadel, A convergent scheme for a non-local coupled system modelling dislocations densities dynamics, Math. Comp., 77 (2008), pp. 789-812.

[7] E. B. Fabes and N. Garofalo, Mean value properties of solutions to parabolic equations with variable coefficients, J. Math. Anal. Appl., 121 (1987), pp. 305-316.

[8] S. Forest and R. SedláČek, Plastic slip distribution in two-phase laminate microstructures: dislocation based versus generalized continuum approaches, Philosophical Magazine, 83 (2003), pp. 245-276.

[9] D. François, A. Pineau, and A. Zaoui, Comportement mécanique des matériaux, vol. volume I : élasticité plasticité (1991), volume II : visco-plasticité, endommagement, mécanique de la rupture, mécanique du contact (1993), Hermès.

[10] N. Garofalo and E. Lanconelli, Asymptotic behavior of fundamental solutions and potential theory of parabolic operators with variable coefficients, Math. Ann., 283 (1989), pp. 211-239.

[11] I. Groma And P. Balogh, Investigation of dislocation pattern formation in a two dimentional selfconsistent field approximation, Acta Mater, 47 (1999), pp. 3647-3654.

[12] I. Groma, F. F. Czikor, And M. ZAiser, Spatial correlations and higher-order gradient terms in a continuum description of dislocation dynamics, Acta Mater, 51 (2003), pp. 1271-1281.

[13] J. R. Hirth And L. Lothe, Theory of dislocations, Second edition, Kreiger publishing company, Florida 32950, 1982.

[14] H. Ibrahim, Analysis of parabolic/Hamilton-Jacobi systems modelizing the dynamics of dislocation densities in bounded domain, Ph.D. thesis.

[15] —_, Existence and uniqueness for a non-linear parabolic/Hamilton-Jacobi coupled system describing the dynamics of dislocation densities, to appear in Ann. Inst. H. Poincaré Anal. Non Linéaire, (2007).

[16] H. Ibrahim, M. Jazar, and R. Monneau, Dynamics of dislocation densities in a bounded channel. Part I: smooth solutions to a singular coupled parabolic system, preprint, hal-00281487.

[17] A. Kufner, O. John, And S. Fučík, Function spaces, Noordhoff International Publishing, Leyden, 1977. Monographs and Textbooks on Mechanics of Solids and Fluids; Mechanics: Analysis.

[18] O. A. Ladyženskaja, V. A. Solonnikov, and N. N. Ural'Ceva, Linear and quasilinear equations of parabolic type, Translated from the Russian by S. Smith. Translations of Mathematical Monographs, Vol. 23, American Mathematical Society, Providence, R.I., 1967.

[19] G. M. Lieberman, Second order parabolic differential equations, World Scientific Publishing Co. Inc., River Edge, NJ, 1996. 